\title{
WinProphet: a user-friendly pipeline management system for proteomics data analysis based on Trans-Proteomic Pipeline
}

Ching-Tai Chen ${ }^{1, *}$, , Chu-Ling Ko ${ }^{2, \ddagger}$, Wai-Kok Choong ${ }^{1, \ddagger}$, Jen-Hung Wang ${ }^{1}$, Wen-Lian Hsu ${ }^{1}$, and Ting-Yi Sung ${ }^{1 *}$

${ }^{1}$ Institute of Information Science, Academia Sinica, Nankang, Taipei 115, Taiwan

${ }^{2}$ Department of Computer Science, National Chiao Tung University, Hsinchu 300, Taiwan

*To whom correspondence should be addressed.

The authors wish it to be known that, in their opinion, the first three authors should be regarded as Joint First Authors.

\section{Supporting Information}

Text S1. XML schema of the pipeline...

Figure S1. Screenshot of Report Generator showing identification results.

Figure S2. Flowchart and screenshot of the first pipeline.

Figure S3. Flowchart and screenshot of the third pipeline.

Figure S4. Flowchart and screenshot of the fourth pipeline. 8

Figure S5. Flowchart and screenshot of the fifth pipeline. 9

Table S1. The number of identified proteins, peptides, and PSMs for the five pipelines 10 
Text S1. XML schema of the pipeline

$<$ ?xml version="1.0" encoding="UTF-8" ?>

<xs:schema xmlns:xs="http://www.w3.org/2001/XMLSchema">

$<x s$ :element name="winprophet" $>$

$<x$ :complexType $>$

$<x$ s:sequence>

$<x$ :element name="function" type="xs:string"/>

$<x s: e l e m e n t$ name="input">

$<x s$ :complexType>

$<x$ :sequence $>$

$<x$ :element name="dirName" type="xs:string"/>

$<x$ :element name="inputFile" type="xs:string"/>

$<x$ :element name="outputFile" type="xs:string"/>

$<x$ :element name="functionType" type="xs:string"/>

$</ x s$ :sequence $>$

$</ x s$ :complexType $>$

$</ x s$ :element $>$

<xs:element name="item" maxOccurs="unbounded">

$<x$ :complexType>

$<x$ s:sequence>

$<x s$ :element name="ReportGenerator" type="xs:string"/>

$<x s$ :element name="pepXML" type="xs:string"minOccurs="0"/>

$<x s:$ element name="protXml" type="xs:positivelnteger"/>

$<x s$ :element name="mayuCsv" type="xs:decimal"/>

$</ x s$ :sequence $>$

$</ x s$ :complexType $>$

$</ x s$ :element $>$

$<x$ :element name="item" maxOccurs="unbounded">

$<x$ :complexType>

$<x s$ :sequence $>$

<xs:element name="UserCommand" type="xs:string"/>

$<x$ :element name="commandStr" type="xs:string"minOccurs="0"/>

$</ x s$ :sequence $>$

$</ x s$ :complexType $>$

$</ x s$ :element $>$

$</ x s$ :sequence $>$

$<x s:$ attribute name="orderid" type="xs:string"use="required"/>

$</ x s$ :complexType>

$</ x s$ :element>

$</ x s$ :schema $>$ 


\section{Figure S1.}

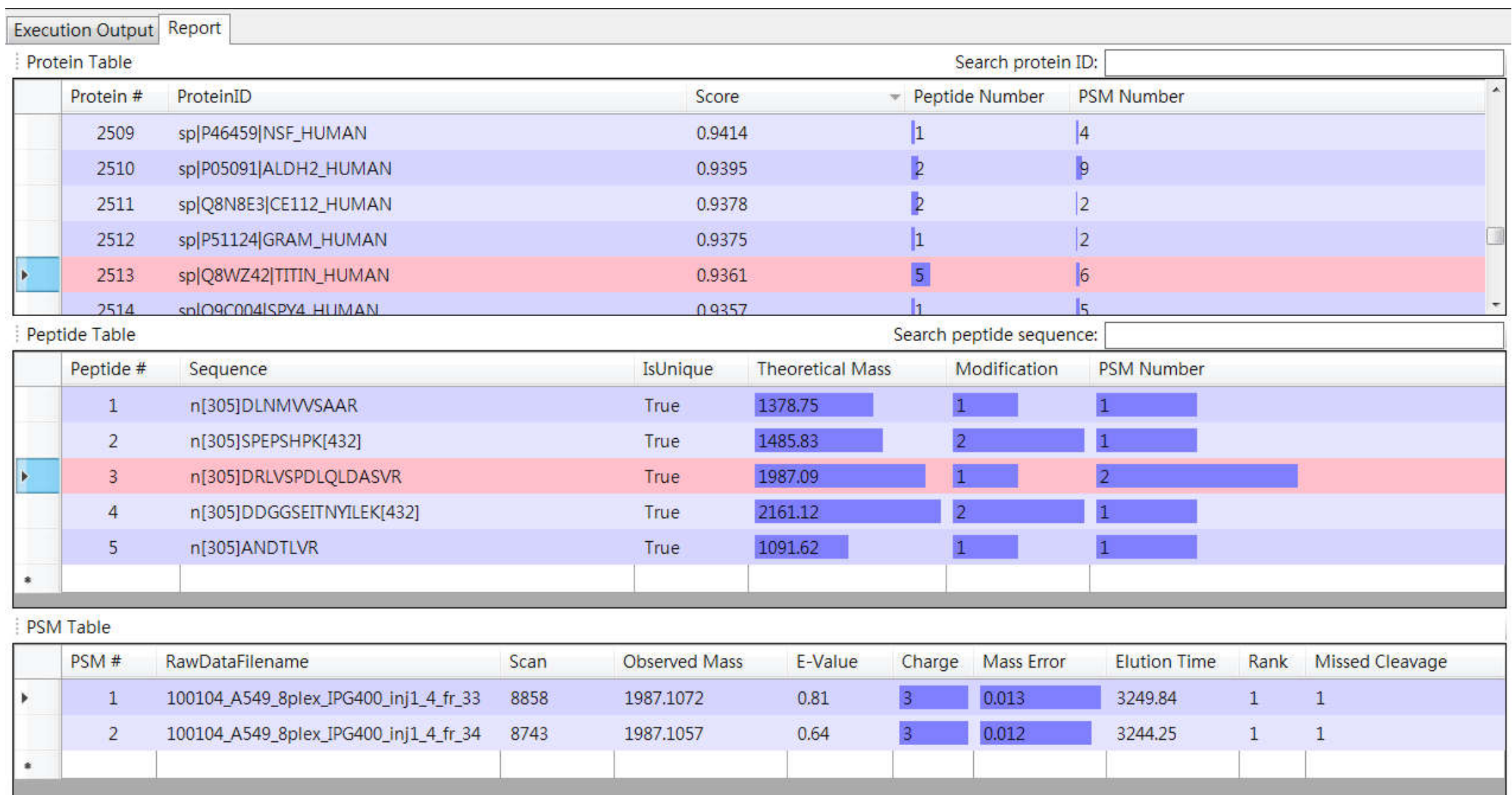

Figure S1. Screenshot of Report Generator showing identification results. The user interface of Report Generator consists of three components, a protein table, a peptide table, and a PSM table. PSM table lists all the PSMs of the selected peptide; peptide table lists all the peptides of the selected protein. All of the proteins and peptides listed in the tables satisfy the criterion of $1 \%$ false discovery rate. Score column in protein table denotes the ProteinProphet probability assigned to each protein group. If users run Mayu instead or only import a pep.xml file to Report Generator, the column will be hidden. 


\section{Figure S2.}

A

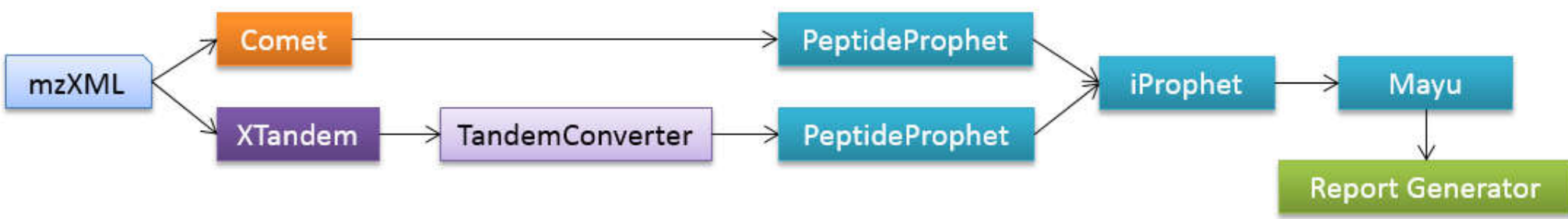

\begin{tabular}{|c|c|c|c|c|c|c|c|}
\hline \multicolumn{7}{|c|}{ WinProphet } & 一回 \\
\hline \multicolumn{8}{|c|}{ 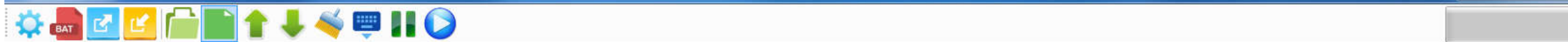 } \\
\hline Status & Directory & Input & Function & & Output & Param & Remove \\
\hline & C:ITPPIdatalerwinia_carotovora & TMT_Erwinia_1uLSike_Top10HCD_isol2_45stepped_60min_01.... & Comet & - & TMT_Erwinia_1uLSike_Top10HCD_i... & parameter & remove \\
\hline & C:ITPPIdatalerwinia_carotovora & TMT_Erwinia_luLSike_Top10HCD_isol2_45stepped_60min_01.... & $\mathrm{X}$ !Tandem & 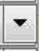 & TMT_Erwinia_1uLSike_Top10HCD_i... & parameter & remove \\
\hline & C:ITPPIdatalerwinia_carotovora & TMT_Erwinia_1uLSike_Top10HCD_isol2_45stepped_60min_01.t... & Tandem Converter & + & TMT_Erwinia_1uLSike_Top10HCD_i... & parameter & remove \\
\hline & C:ITPPIdatalerwinia_carotovora & TMT_Erwinia_1uLSike_Top10HCD_isol2_45stepped_60min_01.p... & PeptideProphet & - & pp_comet.pep.xml & parameter & remove \\
\hline & C:ITPPIdatalerwinia_carotovora & TMT_Erwinia_luLSike_Top10HCD_isol2_45stepped_60min_01_t.... & PeptideProphet & 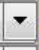 & pp_tandem.pep.xml & parameter & remove \\
\hline & C:ITPPIdatalerwinia_carotovora & pp_comet.pep.xml,pp_tandem.pep.xml & iProphet & 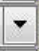 & ip_pp_erwinia.ipro.pep.xml & parameter & remove \\
\hline & C:ITPPIdatalerwinia_carotovora & ip_pp_erwinia.ipro.pep.xml & Mayu & $\checkmark$ & Mayu_ip_pp_erwinia_FDR.csv & parameter & remove \\
\hline & C:ITPPIdatalerwinia_carotovora & ip_pp_erwinia.ipro.pep.xml,Mayu_ip_pp_erwinia_FDR.csv & Report Generator & 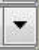 & & parameter & remove \\
\hline
\end{tabular}

Figure S2. Flowchart and screenshot of the first pipeline. (A) The flowchart illustrates protein identification for a single $\mathrm{mzXML}$ file of an Erwinia sample. (B) Screenshot of the pipeline editor shows a total of 8 operations as well as their input and output files. 


\section{Figure S3.}

A

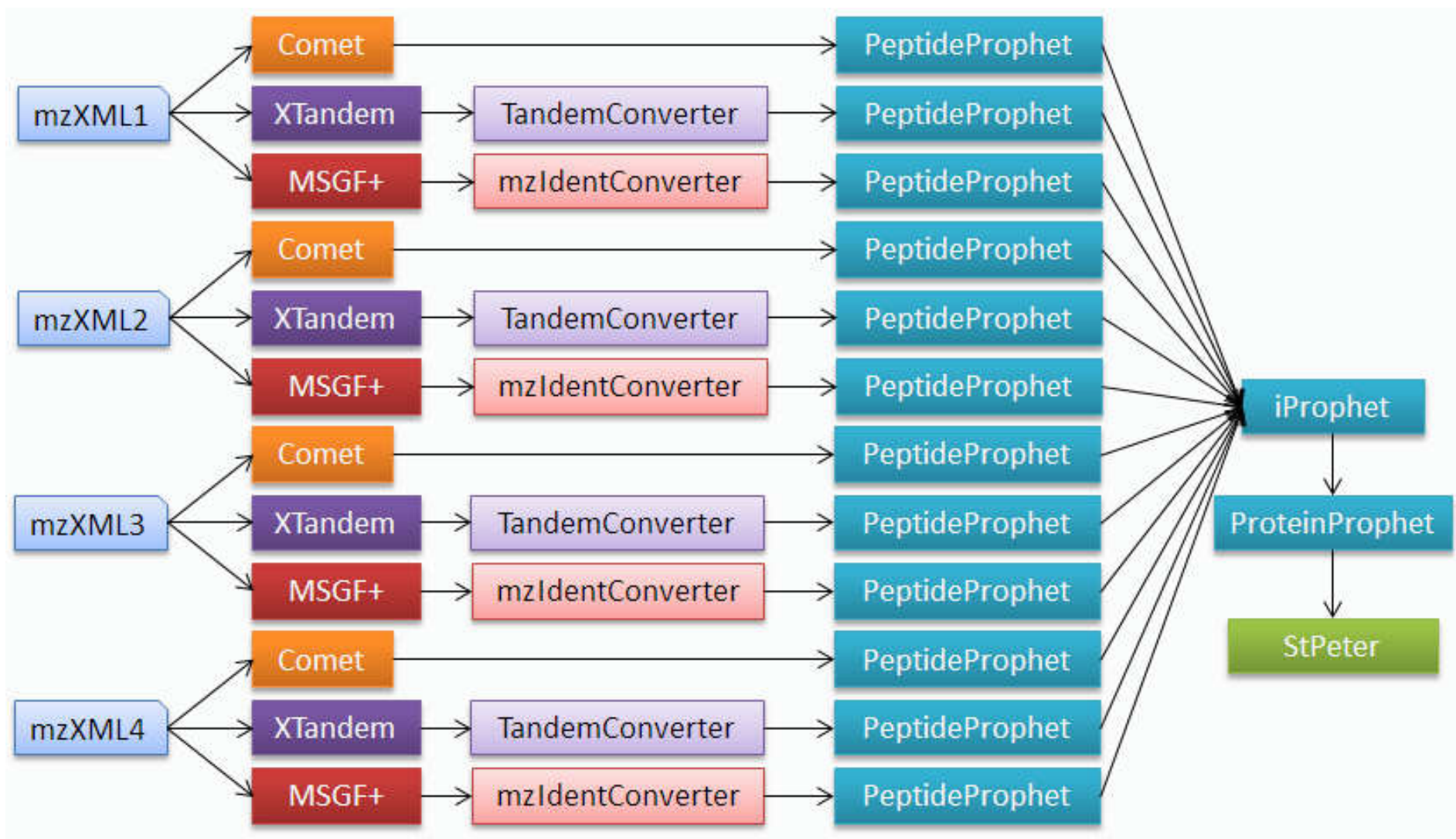




\begin{tabular}{|c|c|c|}
\hline Status & Directory & Input \\
\hline & C:ITPPIdatalmcf7_lfq & MCF7_phos_DDA_1.mzXML \\
\hline & C:ITPPIdatalmof7_lfg & MCF7_phos_DDA_2.mzXML \\
\hline & C:ITPPIdatalmcf7_lfq & MCF7_phos_DDA_3.mzXML \\
\hline & C:ITPPidatalmcf7_lfq & MCF7_phos_DDA_4.mzXML \\
\hline & C:ITPPIdatalmcf7_lfq & MCF7_phos_DDA_1.mzXML \\
\hline & C:ITPPIdatalmcf7_lfq & MCF7_phos_DDA_2.mzXML \\
\hline & C:ITPPIdatalmcf7_lfq & MCF7_phos_DDA_3.mzXML \\
\hline & C:ITPPIdatalmcf7_lfq & MCF7_phos_DDA_4.mzXML \\
\hline & C:ITPPIdatalmcf7_lfq & MCF7_phos_DDA_1.tandem \\
\hline & C:ITPPIdatalmcf7_lfq & MCF7_phos_DDA_2.tandem \\
\hline & C:ITPPIdatalmcf7_lfq & MCF7_phos_DDA_3.tandem. \\
\hline & C:ITPPIdatalmcf7_lfq & MCF7_phos_DDA_4ttandem \\
\hline & C:ITPPIdatalmcf7_lfq & MCF7_phos_DDA_1.mzid \\
\hline & C:ITPPIdatalmcf7_lfg & MCF7_phos_DDA_2.mzid \\
\hline & C:ITPPIdatalmcf7_lfq & MCF7_phos_DDA_3.mzid \\
\hline & C:ITPPIdatalmof7_lfq & MCF7_phos_DDA_4.mzid \\
\hline & C:ITPPIdatalmcf7_lfq & MCF7_phos_DDA_1.mzXML \\
\hline & C:ITPPIdatalmcf7_lfq & MCF7_phos_DDA_2mzXML \\
\hline & C:ITPPidatalmcf7_lfq & MCF7_phos_DDA_3mzXML \\
\hline & C:ITPPIdatalmcf7_lfq & MCF7_phos_DDA_4.mzXML \\
\hline & C:ITPPIdatalmcf7_lfq & MCF7_phos_DDA_1_tandem.pep.xml \\
\hline & C:ITPPIdatalmcf7_lfq & MCF7_phos_DDA_2_tandem.pep.xml \\
\hline & C:ITPPIdatalmcf7_lfq & MCF7_phos_DDA_3_tandem.pep.xml \\
\hline & C:ITPPIdatalmcf7_lfq & MCF7_phos_DDA_4_tandem.pep.xml \\
\hline & C:ITPPIdatalmcf7_lfq & MCF7_phos_DDA_1.pepXML \\
\hline & C:ITPPIdatalmcf7_lfq & MCF7_phos_DDA_2.pepXML \\
\hline & C:ITPPIdatalmcf7_lfq & MCF7_phos_DDA_3.pepXML \\
\hline & C:ITPPIdatalmcf7_lfq & MCF7_phos_DDA_4.pepXML \\
\hline & C:ITPPIdatalmcf7_lfq & MCF7_phos_DDA_1.pep.xml \\
\hline & C:ITPPIdatalmcf7_lfq & MCF7_phos_DDA_2.pep.xml \\
\hline & C:ITPPIdatalmcf7_lfq & MCF7_phos_DDA_3.pep.xml \\
\hline & C:ITPPIdatalmcf7_lfq & MCF7_phos_DDA_4.pep.xml \\
\hline & C:ITPPIdatalmcf7_lfq & pp_t_1.pep.xml,pp_t_2.pep.xml,pp_t_3.pep.xml,pp_t_4.pep.xml,pp_m_1.pep.xml,pp_m_2.pep.xml,pp_m_3.... \\
\hline & C:ITPPIdatalmcf7_lfq & ip_pp_4t4m4c.ipro.pep.xml \\
\hline
\end{tabular}

\begin{tabular}{|c|c|c|c|c|}
\hline Function & & Output & Param & Remove \\
\hline $\mathrm{X} !$ Tandem & $\checkmark$ & MCF7_phos_DDA_1.tandem & parameter & remove \\
\hline X!Tandem & $\checkmark$ & MCF7_phos_DDA_2.tandem & parameter & remove \\
\hline X!Tandem & $\checkmark$ & MCF7_phos_DDA_3.tandem & parameter & remove \\
\hline X!Tandem & 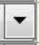 & MCF7_phos_DDA_4tandem & parameter & remove \\
\hline MSGF+ & $\checkmark$ & MCF7_phos_DDA_1.mzid & parameter & remove \\
\hline MSGF+ & $\checkmark$ & MCF7_phos_DDA_2.mzid & parameter & remove \\
\hline MSGF+ & 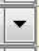 & MCF7_phos_DDA_3.mzid & parameter & remove \\
\hline MSGF+ & 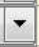 & MCF7_phos_DDA_4.mzid & parameter & remove \\
\hline Tandem Converter & - & MCF7_phos_DDA_1_tandem.pep.xml & parameter & remove \\
\hline Tandem Converter & 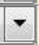 & MCF7_phos_DDA_2_tandem.pep.xml & parameter & remove \\
\hline Tandem Converter & 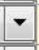 & MCF7_phos_DDA_3_tandem.pep.xml & parameter & remove \\
\hline Tandem Converter & $\checkmark$ & MCF7_phos_DDA_4_tandem.pep.xml & parameter & remove \\
\hline mzIdent Converter & $\checkmark$ & MCF7_phos_DDA_1.pepXML & parameter & remove \\
\hline mzIdent Converter & 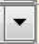 & MCF7_phos_DDA_2pepXML & parameter & remove \\
\hline mzIdent Converter & 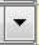 & MCF7_phos_DDA_3pepXML & parameter & remove \\
\hline mzIdent Converter & 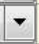 & MCF7_phos_DDA_4pepXML & parameter & remove \\
\hline Comet & $\checkmark$ & MCF7_phos_DDA_1.pep.xml & parameter & remove \\
\hline Comet & 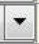 & MCF7_phos_DDA_2.pep.xml & parameter & remove \\
\hline Comet & $\checkmark$ & MCF7_phos_DDA_3.pep.xml & parameter & remove \\
\hline Comet & $\checkmark$ & MCF7_phos_DDA_4,pep.xml & parameter & remove \\
\hline PeptideProphet & - & pp_t_1.pep.xml & parameter & remove \\
\hline PeptideProphet & 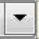 & pp_t_2.pep.xml & parameter & remove \\
\hline PeptideProphet & - & pp_t_3.pep.xml & parameter & remove \\
\hline PeptideProphet & - & pp_t_4.pep.xml & parameter & remove \\
\hline PeptideProphet & - & pp_m_1.pep.xml & parameter & remove \\
\hline PeptideProphet & - & pp_m_2.pep.xml & parameter & remove \\
\hline PeptideProphet & 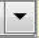 & pp_m_3.pep.xml & parameter & remove \\
\hline PeptideProphet & - & pp_m_4.pep.xml & parameter & remove \\
\hline PeptideProphet & - & pp_c_1.pep.xml & parameter & remove \\
\hline PeptideProphet & 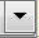 & pp_c_2.pep.xml & parameter & remove \\
\hline PeptideProphet & - & pp_c_3.pep.xml & parameter & remove \\
\hline PeptideProphet & - & pp_c_4.pep.xml & parameter & remove \\
\hline iProphet & 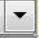 & ip_pp_4t4m4c.ipro.pep.xml & parameter & remove \\
\hline ProteinProphet & - & prot_ip_pp_4t4m4c.prot.xml & parameter & remove \\
\hline User Command & - & & parameter & remove \\
\hline
\end{tabular}

Figure S3. Flowchart and screenshot of the third pipeline. (A) The flowchart illustrates identification and label-free quantitation of 4 mzXML files from MCF-7 breast cancer cell lines. (B) Screenshot of the pipeline editor shows a total of 35 operations as well as their input and output files, where label-free quantitation using StPeter is expressed in the User Command function. 


\section{Figure S4.}

A

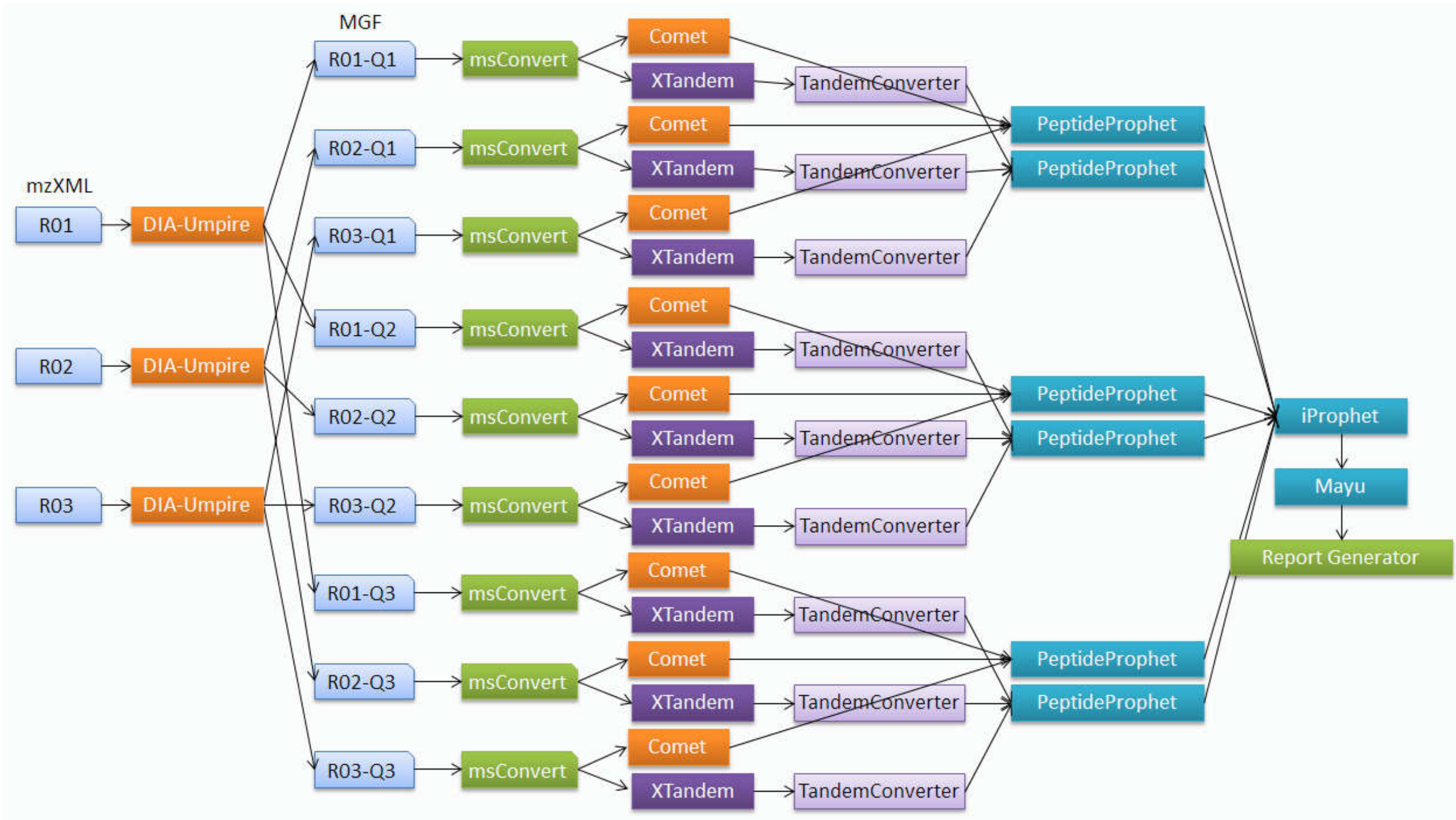




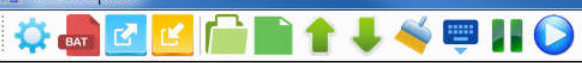

\begin{tabular}{|c|c|c|}
\hline Status & Directory & Input \\
\hline & & \\
\hline & C:ITPPIdata Hela 1ug & Hela1ų_DIA_10Da_150226_01_Q1.mzXXVL \\
\hline & C:ITPPIdatalHelalug & Hela1ug_DIA_10Da_150226_01_Q2.mzXVLL \\
\hline & C:ITPPIdatalHela1ug & Hela1ug_DIA_10Da_150226_01_Q3.mzXML \\
\hline & C:ITPPIdatalHela1ug & Hela1ug_DIA_10Da_150226_02_Q1.mzXML \\
\hline & C:ITPPIdatalHelalug & Hela1ug_DIA_10Da_150226_02_Q2.mzXML \\
\hline & C:ITPPIdatalHelalug & Hela1ug_DIA_10Da_150226_02_Q3.mzXML \\
\hline & C:ITPPIdatalHelalug & Hela1ug_DIA_10Da_150226_03_Q1.mzXNML \\
\hline & C:ITPPIdatalHela1ug & Hela1ug_DIA_10Da_150226_03_Q2.mzXVIL \\
\hline & C:ITPPIdatalHela1ug & Hela1ug_DIA_10Da_150226_03_Q3.mzXML \\
\hline & C:ITPPIdatalHelalug & Hela1ug_DIA_10Da_150226_01_Q1.mzXML \\
\hline & C:ITPPIdatalHelalug & Hela1ug__DIA_10Da_150226_01_Q2.mzXML \\
\hline & C:ITPPIdatalHelalug & Hela1ug_DIA_10Da_150226_01_Q3.mzXML \\
\hline & C:ITPPIdatalHela1ug & Hela1u__DIA_10Da_150226_02_Q1.mzXML \\
\hline & C:ITPPIdatalHelalug & Hela1ug__DIA_10Da_150226_02_Q2.mzXML \\
\hline & C:ITPPIdatalHela1ug & Hela1ug_DIA_10Da_150226_02_Q3.mzXML \\
\hline & C:ITPPIdatalHelalug & Hela1ug_DIA_10Da_150226_03_Q1.mzXML \\
\hline & C:ITPPIdatal Hela Iug & Hela1ug_DIA_10Da_150226_03_Q2.mzXML \\
\hline & C:ITPPIdatalHela1ug & Hela1ug_DIA_10Da_150226_03_Q3.mzXML \\
\hline & C:ITPPIdatalHelalug & Hela1ug_DIA_10Da_150226_01_Q1.tandem \\
\hline & C:ITPPIdatalHelalug & Hela1ug_DIA_10Da_150226_01_Q2.tandem \\
\hline & C.ITPPIdatalHela1ug & Hela1ug_DIA_10Da_150226_01_Q3.tandem \\
\hline & C:ITPPIdatalHelalug & Hela1ug_DIA_10Da_150226_02_Q1.tandem \\
\hline & C:ITPPIdatalHela1ug & Hela1ug_DIA_10Da_150226_02_Q2.tandem \\
\hline & C:ITPPIdatalHelalug & Hela1ug_DIA_10Da_150226_02_Q3.tandem \\
\hline & C:ITPPIdatalHelalug & Hela1ug_DIA_10Da_150226_03_Q1.tandem \\
\hline & C:ITPPIdatalHelalug & Hela1ug_DIA_10Da_150226_03_Q2.tandem \\
\hline & C:ITPPIdatalHelalug & Hela1ug_DIA_10Da_150226_03_Q3.tandem \\
\hline & C:ITPPIdatalHela1ug & Hela1ug_DIA_10Da_150226_01_Q1.pep.xml,Hela1ug_DIA_10D \\
\hline & C:ITPPIdatalHelalug & Hela1ų_DIA_10Da_150226_01_Q2.pep.xml,Hela1ug_DIA_10D \\
\hline & C:ITPPIdatalHelalug & Hela 1ug_DIA_10Da_150226_01_Q3.pep.xml,Hela1ug_DIA_10D \\
\hline & C:ITPPIdatalHela lug & Hela1ug_DIA_10Da_150226_01_Q1_tandem.pep.xm1,Hela lug_L \\
\hline & C:ITPPIdatalHelalug & Hela 1ug_DIA_10Da_150226_01_Q2_tandem.pep.xml,Hela1ug_D \\
\hline & C:ITPPIdatalHelalug & Hela 1ų_DIA_10Da_150226_01_Q3_tandem,pep.xml,Hela1ug_D \\
\hline & C:ITPPIdatalHelalug & 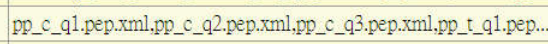 \\
\hline & C:ITPPIdatalHelalug & ip_pp_hela.ipro.pep.xml \\
\hline & C:ITPPIdatalHela1ug & ip_pp_hela.ipro.pep.xml,Mayu_ip_pp_hela_FDR.csv \\
\hline
\end{tabular}

\begin{tabular}{|c|c|c|c|c|}
\hline Function & & Output & Param & Remove \\
\hline User Command & - & & parameter & remove \\
\hline User Command & - & C:ITPPIdatalHelalugIHela1ug_DIA_... & parameter & remove \\
\hline X!Tandem & -1 & Hela1ug_DIA_10Da_150226_01_Q1.t... & parameter & remove \\
\hline $\mathrm{X}$ !Tandem & -1 & Hela1ug_DIA_10Da_150226_01_Q2.t... & parameter & remove \\
\hline $\mathrm{X}$ !Tandem & -1 & Hela1ug_DIA_10Da_150226_01_Q3.t... & parameter & remove \\
\hline X!Tandem & -1 & Hela1ug_DIA_10Da_150226_02_Q1.t... & parameter & remove \\
\hline $\mathrm{X!Tandem}$ & -1 & Helalug_DIA_10Da_150226_02_Q2.t.... & parameter & remove \\
\hline X!Tandem & -1 & Hela1ug_DIA_10Da_150226_02_Q3.t... & parameter & remove \\
\hline $\mathrm{X!Tandem}$ & -1 & Hela1ug_DIA_10Da_150226_03_Q1.t... & parameter & remove \\
\hline $\mathrm{X}$ !Tandem & -1 & Hela1ug_DIA_10Da_150226_03_Q2.t... & parameter & remove \\
\hline X!Tandem & -1 & Hela1ug_DIA_10Da_150226_03_Q3.t... & parameter & remove \\
\hline Comet & -1 & Hela1ug_DIA_10Da_150226_01_Q1.... & parameter & remove \\
\hline Comet & -1 & Hela1ug_DIA_10Da_150226_01_Q2.... & parameter & remove \\
\hline Comet & -1 & Hela1ug_DIA_10Da_150226_01_Q3.... & parameter & remove \\
\hline Comet & -1 & Hela1ug_DIA_10Da_150226_02_Q1.... & parameter & remove \\
\hline Comet & -1 & Hela1ug_DIA_10Da_150226_02_Q2.... & parameter & remove \\
\hline Comet & -1 & Hela1ug_DIA_10Da_150226_02_Q3.... & parameter & remove \\
\hline Comet & -1 & Hela1ug_DIA_10Da_150226_03_Q1.... & parameter & remove \\
\hline Comet & -1 & Hela1ug_DIA_10Da_150226_03_Q2.... & parameter & remove \\
\hline Comet & -1 & Hela1ug_DIA_10Da_150226_03_Q3.... & parameter & remove \\
\hline Tandem Converter & $=1$ & Hela1ug_DIA_10Da_150226_01_Q1_... & parameter & remove \\
\hline Tandem Converter & -1 & Hela1ug_DIA_10Da_150226_01_Q2_... & parameter & remove \\
\hline Tandem Converter & -1 & Hela1ug_DIA_10Da_150226_01_Q3_... & parameter & remove \\
\hline Tandem Converter & -1 & Hela1ug_DIA_10Da_150226_02_Q1_... & parameter & remove \\
\hline Tandem Converter & -1 & Hela1ug_DIA_10Da_150226_02_Q2_... & parameter & remove \\
\hline Tandem Converter & -1 & Hela1ug_DIA_10Da_150226_02_Q3_... & parameter & remove \\
\hline Tandem Converter & -1 & Hela1ug_DIA_10Da_150226_03_Q1_... & parameter & remove \\
\hline Tandem Converter & -1 & Hela1ug_DIA_10Da_150226_03_Q2_... & parameter & remove \\
\hline Tandem Converter & -1 & Hela1ug_DIA_10Da_150226_03_Q3_... & parameter & remove \\
\hline PeptideProphet & 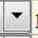 & op_c_q1.pep.xml & parameter & remove \\
\hline PeptideProphet & - & op_c_aq2.pep.rml & parameter & remove \\
\hline PeptideProphet & - & op_c_a3.pep.xml & parameter & remove \\
\hline PeptideProphet & 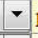 & op_t_q1.pep.xml & parameter & remove \\
\hline PeptideProphet & 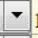 & op_t_q2.pep.xml & parameter & remove \\
\hline PeptideProphet & 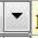 & op_t_a3.pep.xml & parameter & remove \\
\hline iProphet & - & p.pp_hela.ipropep.xml & parameter & remove \\
\hline Мауи & - & Mayu__ip_pp_hela_FDR.csv & parameter & remove \\
\hline Report Generator & - & & parameter & remove \\
\hline
\end{tabular}

Figure S4. Flowchart and screenshot of the fourth pipeline. (A) The flowchart illustrates DIA data analysis of 3 mzXML files of human HeLa cell lysate. (B) Screenshot of the pipeline editor shows a total of 48 operations as well as their input and output files, where the 3 operations of using DIA-Umpire and the 9 operations to perform msConvert are inclusively expressed by the two respective User Command functions. 


\section{Figure S5.}

A

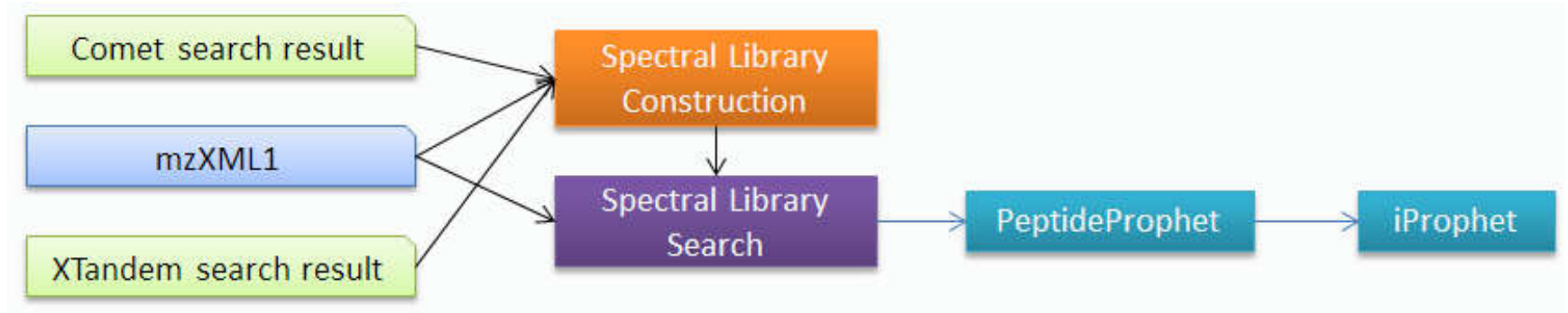

B

\begin{tabular}{|c|c|c|c|c|c|c|c|}
\hline \multicolumn{8}{|c|}{ 囁 WinProphet } \\
\hline \multicolumn{8}{|c|}{ 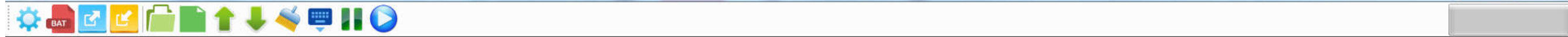 } \\
\hline & Status & Directory & Input & Function & Output & Param & Remove \\
\hline \multirow[t]{4}{*}{ - } & & & & User Command & -1 & parameter & remove \\
\hline & & & & User Command & - C:ITPPIdatalerwinia_libraryConstructionITMT_Erwinia__luLSike_Top10HCD_isol2_... & parameter & remove \\
\hline & & C:ITPPIdatalerwinia_libraryConstruction & TMT_Erwinia_1uLSike_Top10HCD_isol2_45stepped_60min_01.pep.xml & PeptideProphet & - pp_spectrast.pep.xml & parameter & remove \\
\hline & & C:ITPPIdatalerwinia_libraryConstruction & pp_spectrast.pep.xml & iProphet & - ip_pp_spectrast.ipro.pep.xml & parameter & remove \\
\hline * & & & & & - & & \\
\hline
\end{tabular}

Figure S5. Flowchart and screenshot of the fifth pipeline. (A) The flowchart illustrates using the search results and the $m z X M L$ file from the first pipeline to perform spectral library construction and search using SpectraST, followed by statistical validation of search results. (B) A screenshot of the pipeline editor shows a total of 4 operations as well as their input and output files, where the operations of spectral library construction and search using SpectraST are expressed by two User Command functions. The detailed commands are provided in User Guide. 
Table S1. The numbers of identified proteins, peptides, and PSMs for the five pipelines

\begin{tabular}{lrrrr} 
& Validation & Protein Number & Peptide Number & PSM Number \\
\hline Pipeline 1 & 1\% FDR at peptide and protein level & 455 & 1984 & 2504 \\
Pipeline 2 & 1\% FDR at peptide and protein level & 2485 & 4546 & 20484 \\
Pipeline 3 & 1\% FDR at peptide and protein level & 2716 & 9363 & 26624 \\
Pipeline 4 & 1\% FDR at peptide and protein level & 3878 & 23812 & 72270 \\
Pipeline 5 & 1\% FDR at peptide level & 443 & 1701 & 2477 \\
\hline
\end{tabular}

